\title{
A critical take on the World Health Organization's new 'test and treat' strategy: making a case for resource-constrained countries
}

\author{
Fritz Ngale Ilongo, Francis Rakotsoane \\ National University of Lesotho, Roma, Lesotho
}

\begin{abstract}
This article aims to critically explore limitations, which resource-constrained countries like Lesotho face in the sustainable implementation of the WHO 'test and treat' comprehensive strategy for eliminating HIV and AIDS. The methodology is critical thinking and qualitative theoretical analyses, articulated around the UNAIDS 90-90-90 strategy. The researchers argue that the context of Lesotho involves serious human, technical, and infrastructural challenges, which may hamper the efficient and effective implementation of the 'test and treat' strategy. In line with the preceding, they suggest that the country is among the poorest in the world, and faces severe shortage of conventional health workers and professional counsellors. The paper's recommendation as a way forward: widespread and timely access to treatment, extensive countrywide testing, training and deployment of social work and counselling professionals, mass education and sensitization, development and sustenance of linkages to care and patient follow-up on referrals, putting in place retention in care mechanisms, optimization of every component of 'test and treat', and trying out alternative treatment models like the Anti-Retroviral Treatment and Access to Services (ARTAS).
\end{abstract}

HIV AIDS Rev 2017; 16, 3: 135-139 DOI: https://doi.org/10.5114/hivar.2017.68022

Key words: 'test and treat' strategy, UNAIDS 90-90-90 strategy, Anti-Retroviral Treatment and Access to Services.

\section{Introduction}

The world of human immunodeficiency virus/acquired immune deficiency syndrome (HIV/AIDS) has changed dramatically over the last 35 years. It was a blow to humanity when first described in 1981 as it was an inevitably fatal disease. The disease was expected to devastate the world in an unprecedented manner. In 1996, there was the first glimmer of hope when highly active antiretroviral treatment (HAART) was shown to suppress the viral replication significantly and improve survival [1].

Address for correspondence: Dr. Fritz Ngale Ilongo, National University of Lesotho, PO Box 180, Roma, Lesotho, e-mail: cactuso2001@yahoo.com

After the discovery of HAART, it took the world some time to know exactly when to start treatment. The precise time as to when to start the antiretroviral treatment was generally left to the discretion of the medical personnel in individual countries. As late as 2008, the optimal time to be put on antiretroviral treatment still remained uncertain $[2,3]$. With the improved knowledge of HIV and AIDS by the health sector in general, however, more definite proposals were made including putting anyone whose CD4 count dropped to 350 cells $/ \mathrm{mm}^{3}$ on antiretroviral treatment prior

Article history:

Received: 28.10 .2016

Received in revised form: 16.02.2017

Accepted: 27.02.2017

Available online: 25.05 .2017
International Journal of HIV-Related Problems

HIV \& AIDS

R e v i e w 
to 2013, and having on treatment all people with the CD4 of 500 cells $/ \mathrm{mm}^{3}$ (WHO 2015) or worse. As more and more scientific facts on HIV and AIDS became available, there appeared to be a steady move to starting the antiretroviral treatment as early as possible [3, 4].

After long and sometimes controversial debates on the issue by scholars in various parts of the world, the World Health Organization (WHO) has, as from 30 September 2015 , provided new guidelines, in which it is now recommending the antiretroviral treatment for everyone who tests HIV positive (WHO 2015). What this means essentially, is that all debates on when to start antiretroviral treatment have now been put to rest. This has generally been accepted not as good news by many people and international agents including the UNAIDS, which had already set itself an ambitious target of ending the AIDS epidemic by 2030 [5]. To achieve this target, the UNAIDS launched a strategy termed $90-90-90$. This targeted that by $2020,90 \%$ of all people living with HIV should be aware of their HIV status, $90 \%$ of all people with diagnosed HIV infection should be put on sustained antiretroviral therapy, and $90 \%$ of all people receiving antiretroviral therapy should have viral suppression (UNAIDS 2016).

As a result of WHO new guidelines, many countries of the world are today speaking 'test and treat' strategy, by which they mean that all those who test positive for HIV should be immediately put on antiretroviral treatment. While this may and should sound great in the ears of all those who want to see the world being rid of HIV and AIDS, putting everyone who is HIV positive on antiretroviral treatment in resource-constrained countries, without any guarantee that the necessary numbers of trained health workers and the needed ARVs will be sustainably made available at all times and places where they will be needed timeously, is something that needs to be cautiously approached.

This paper is an attempt to explore some of the likely challenges and consequences of 'test and treat' strategy, as applied to resource-constrained countries with special reference to Lesotho. Using critical thinking as its method of approach, the paper will delve into some of the most subtle issues that will have to be expatiated upon, and be well understood and dealt with before such countries can embark on implementing the 'test and treat' strategy, which generally appears to be a good policy that may turn out to be suicidal if implemented without prior consideration of all implications.

\section{The 'test and treat' idea}

'Test and treat' is a comprehensive strategy for eliminating HIV and AIDS by regularly testing people, putting the infected on antiretroviral treatment (ART) in spite of their CD4 level, and care improvement to reduce or prevent transmission of the disease by people living with HIV and AIDS (PLWHA). Research findings seem to confirm the aforementioned premises of test and treat [6].
The test and treat approach is based on two theories [6]: 1) early diagnosis and treatment will decrease the risk of HIV and AIDS, and non-HIV and AIDS-related health problems in people living with HIV and AIDS,

2) reduction of viral load to undetectable levels will dramatically reduce the current transmission rate of HIV.

The test and treat approach has two goals, namely, improvement of the health outcomes in people who do not yet know their serostatus, and the reduction of HIV transmission, based on the assumption that PLWHA who are receiving treatment are far less likely to transmit infection to others [7].

If fully implemented, test and treat programs could help increase the number of people receiving early diagnoses when their immune systems are relatively healthy and, furthermore, could produce a decrease in the number of people unaware of their status who, without HIV treatment, are more likely to infect others. It is clear that test and treat has the potential to save lives.

A growing body of evidence suggests that earlier treatment can extend lives and reduce mortality [8]. A test and treat frame-work would start PLWHA on ART earlier in disease course than the current recommendations, and could have a major influence on the HIV epidemic. As more people with higher CD4 counts began and maintained treatment, infectiousness would decrease. Linkage to and retention in high-quality HIV primary care is essential for the strategy to work [7]. One of the major reasons to support the test and treat approach is simply the effectiveness of ART in reducing HIV transmission. According to one source, even with the phenomena of drug-resistant virus and adherence difficulties, only $15-25 \%$ of PLWHA on treatment have detectable virus [7]. One of the most exciting breakthroughs at the recent International AIDS Society Conference in Rome was a report of the HIV Prevention Trials Network (HPTN) 052 study, showing that HIV-infected men and women who were on ART had a 96\% reduced risk of transmitting the virus to their uninfected sexual partners [9]. This is further confirmed by the PARTNER study results presented during the 2016 Durban AIDS conference, which showed that there is virtually zero risk of HIV transmission in mixed couples with undetectable viral load, after enrolled couples had sex 58,000 times without condoms.

\section{Challenges to implementation of 'test and treat' strategy - the case of Lesotho}

In May 2016, the Prime Minister of Lesotho, Dr. Pakalitha Mosisili, launched the 'test and treat' as the country's new approach to fighting HIV infection. Proudly declaring his country the first one to do this in Africa, the Prime Minister said: 'Preventing future HIV infection by introducing this 'test and treat' approach we can move forward and adapt it for the benefit of all Basotho. This is a very important day in Lesotho as I was told that we are the first country in Africa to launch this initiative of 'test and treat', and we are very 
proud of it, and hope it will make a change in our community' (Informative, 19-25 April 2016: 12).

The country's Minister of Health, Dr. Molotsi Monyamane, indicated that his Ministry was very happy about the launch as the move would help infected persons in Lesotho to be put on treatment while they were still healthy and strong enough to produce their own food. Speaking at the same occasion on behalf of the United States of America, President's Emergency Plan for AIDS Relief (PEPFAR) coordinator, Reuben Heylett, hailed this as a huge step in the right direction towards taking the war to the HIV epidemic, and promised the country a continued support for following the World Health Organization's (WHO) recommendations to improve the lives of Basotho (Informative, 19-25 April 2016: 12).

While adapting this approach has a potential to turn things around in the fight against the HIV pandemic in well-resourced developed countries; the same may not necessarily be said in the context of the resource-constrained countries, given technical and infrastructural challenges usually found in such countries and their general poor track record in managing the AIDS pandemic. What follows below are some of the arguments for which the paper finds the implementation of the 'test and treat' strategy by Lesotho Government a bit premature.

Widespread testing and treatment has large financial cost and other resources implications; as more people are engaged into test and treat, more resources will be needed. Unfortunately, the country's current situation is such that:

It ranks 158 out of 187 countries on the UN Human Development ranking, falling into the category of low human development. National poverty figures indicate that $57.1 \%$ of the population lives below the national poverty line. Income distribution is unequal indicated by a Gini coefficient of 0.54. Due to the devastating impact of the HIV/AIDS epidemic, average life expectancy stands at 48.7 years. Literacy rates are high at $80.9 \%$ for men and $96.9 \%$ for women in the age group of 15-49 years. The much higher literacy rates for women are because women are better represented in the education system, while men tend livestock and engage in subsistence farming at an early age.

The population of the country is 1.9 million with an annual growth rate of $0.9 \%$, an increase of 0.1 percentage points from the $0.8 \%$ in the period 1996-2006. The country is very young demographically, with about $40 \%$ of the population comprising youth (15-35 years). The population is predominantly rural with approximately $77 \%$ residing in rural areas. The rural population depends to a large extent on subsistence agriculture for their livelihoods. However, owing to low agricultural productivity and with only $10 \%$ of its land surface available for arable agriculture, the country relies heavily upon imports from South Africa. Domestic cereal production only satisfies $30 \%$ of Lesotho's needs.

(The Kingdom of Lesotho Millennium Development Goals Status Report 2013)

The above scenario undoubtedly depicts Lesotho as a country confronted by many challenges, which are likely to make it extremely difficult if not impossible for it to sustain- ably fund the massive planned ARVs rollout under the 'test and treat' strategy from its own coffers. To have an adequate funding that is needed to keep a constant supply of the ARVs, the country will need even more intervention of its development partners than it is currently doing with the limited numbers that it supplies with the ARVs. The problem with foreign aid is that its lifespan or sustainability depends on good relationship that exists between the donor and the recipient. Should the relationship between the two suffer any form of strain, the aid suffers also the same strain, at times, to the extent that it may be abruptly stopped for good by the donor. This is not impossible to happen with Lesotho. The country's recent failure to have its AGOA beneficiary status renewed due to its failure to meet certain conditions put as prerequisite to benefitting from AGOA by the US Government is a clear example of such possibility. This has to be understood against the background that a huge chunk of the money Lesotho is currently using to fund its HIV and AIDS programs comes from the US President's Emergency Plan for AIDS Relief (PEPFAR) and Global Fund. In the light of this, putting every citizen who is found HIV positive on ARVs when their government has no capacity to sustainably supply such drugs in the absence of the external financial aid, is nothing short of gambling with people's lives. It is tantamount to committing suicide on the part of those healthy individual citizens who will agree to be put on ARVs without any guarantee from their government that they will never be short of the ARVs supply, regardless of the country's success or failure to secure continued financial aid from its development partners. This too has to be understood against the background that the ARV treatment is a life treatment which, if defaulted, can lead to development of drug resistance that may ultimately lead to one's death.

Another one of Lesotho's current challenges is the problem of food security, which has bedeviled the country as a result of the unfavorable climate change the country has been going through in recent years, and which does not appear to be likely to stop any time soon. It is a known fact that the taking of ARVs goes with eating adequately at the right time in order to facilitate greater effectiveness of administered drugs. Given extremely high unemployment rate with over $50 \%$ of the population living in poverty, with around 30\% living below the poverty line, it will be a great challenge for the government to ensure that all people on ARVs have enough to eat each day (http://eeas.europa.eu/lesotho/index_en.htm). The country has had cases and continues to have cases of people who have abandoned their ARVs treatment as a result of experiencing unbearable corrosive effects of these drugs on their empty stomachs. Three treatment regimens are used in Lesotho. These include the first-line regimen (two nucleoside reverse transcriptase inhibitors [NRTIs] plus one non-nucleoside reverse transcriptase inhibitor [NNRTI]), second-line ART consisting of two NRTIs, but with the addition of a PI, and the following third-line drugs: darunavir (DRV), ritonavir (RTV) as a pharmacokinetic booster, raltegravir (RAL) and etravirine (ETV). 
In addition to the country's gloomy socio-economic situation depicted above, Lesotho has a very poor record of dealing with HIV and AIDS in general. For example, when the country decided to scale up antiretroviral therapy (ART) between 2006 and 2011, it found itself having to resort to the use of 487 lay counsellors (LCs) who were trained to provide HIV testing and counselling (HTC), and ART adherence support due to severe shortage of conventional health workers and professional counsellors. Engaging such lay counsellors helped in increasing the country's HIV testing from 79,394 in 2006 to 274,240 people in 2011, and the number of people on ART from 17,352 to 83,624 within the same period. Despite all the success achieved, the country was not able to retain all the 487 lay counsellors due to a number of reasons including administrative and fiscal barriers that hampered their absorption into the public health system when donor funding declined. This resulted in the number of lay counsellors tumbling down to 165 in 2012, while ART coverage decreased from $61 \%$ to $51 \%$, and facility-based HTC decreased by $15 \%$ from 253,994 in 2011 to 215,042 tests in 2012. The country's rise in HIV statistics is generally attributed to, among other factors, this sad situation. This means that, for resource-constrained countries like Lesotho, a nationally and internationally well-supported counsellor cadre is essential in order to ensure sustainable access to HIV care for all people who test HIV positive [10].

As clearly depicted above, there are obvious several challenges the country is likely to encounter when implementing the 'test and treat' strategy. Recruitment and retention of the necessary number of counsellors is crucial for the success of the strategy. Maintaining supply chains of antiretroviral therapy drugs for the additional numbers of people who are going to be on treatment, when already frequented by non-availability of stocks with fewer numbers of people on treatment, remains the major concern for the strategy's success. Many parts of the country remain inaccessible due to lack of roads. With very few roads due to the country's rough terrain, which creates problems of accessibility to the masses of people living in the rural areas, there exist very few health centers, from which people on ARVs treatment may obtain their supplies. Many of the health centers found in these areas are located far away from villages they are meant to serve. As a result of the long distances that people have to walk before they can reach such centers, many ARVs users default thus jeopardizing their lives. Such defaulting often results in patients developing resistance to treatment. This problem of non-adherence is likely to be compounded when even larger numbers of people who are currently not on treatment are put on it [1]. Details of the three lines of regimens used in Lesotho have been earlier presented. Longterm side effects of medications are also an issue that will need close monitoring by professional health workers. Can the country have enough of or even retain such professionals given the situation in which it is right now? From the above picture of the country's socio-economic conditions, the situation appears gloomy.

\section{What then should be a way forward for a resource-constrained country like Lesotho?}

What then does this mean? Does it mean that the country should ignore the WHO's new guidelines and continue losing its citizens to HIV and AIDS? Not at all. What the paper is arguing for is that the government should see to it that all the necessary resources and infrastructure are in place before it can rush to becoming the first African country to implement the new WHO's HIV fighting strategy, in case it finds itself in a situation that is likely to compromise the general health of its people unnecessarily. First of all, the country should be aware that widespread and timely access to treatment is required for a successful test and treat program. For that reason, there will be a need for an extensive countrywide testing in order to identify people living with HIV and AIDS timeously. For this to happen, the country will need the skills of diverse types of professionals in such fields as health, social work, and counselling to educate people about the importance of this new strategy for their health, and to serve as Linkage Coordinators in general in order to ensure adequate linkages to care and patient follow-up on referrals, so that those diagnosed with HIV do not delay the treatment. This should be done in the understanding that delays affect individual health outcomes, and untreated PLWHA with detectable viral levels are at greater risk of infecting others $[11,12]$.

A host of linkage-to-care models exist; their efficacy varies depending on the population and service context. One such model, which is likely to work well in the context of Lesotho, is what is called Anti-Retroviral Treatment and Access to Services (ARTAS). This is defined as:

(...) an individual-level, multi-session, time-limited intervention with the goal of linking recently diagnosed persons with HIV to medical care soon after receiving their positive test result. ARTAS is based on the Strengths-based Case Management (SBCM) model, which is rooted in Social Cognitive Theory (particularly self-efficacy) and Humanistic Psychology. SBCM is a model that encourages the client to identify and use personal strengths; create goals for himself/herself, and establish an effective, working relationship with the Linkage Coordinator (LC). Linkage Coordinators should have experience providing case management or social services. Ideally, participants should include experienced case managers, social workers, and/ or HIV test counsellors.

(https://effectiveinterventions.cdc.gov/en/highimpactprevention/publichealthstrategies/ARTAS.aspx)

Linking people to care is only half the battle. Once patients are engaged, they must subsequently be retained in care through sustainable supply of the ARVs and the help of the Linkage Coordinators whose responsibilities include, among others, ensuring that no one defaults from the treatment. Every component of test and treat strategy must be optimized if the strategy is to be truly effective. The existing health care infrastructure presents resource challenges, even where model programs for people with HIV have been 
created. Provider and patient education is critical in every component of test and treat. As clinicians and other Linkage Coordinators who began providing HIV/AIDS care at the start of the treatment retire, there will be a need for new ones to replace them. To have a constant supply of needed resources will require substantial money and careful government planning to ensure success of the envisaged strategy. All these will have to be planned carefully by the Government, if the envisaged strategy is to succeed.

\section{Conclusions}

The 'test and treat' strategy, which aims at starting the antiretroviral treatment of all people who have tested HIV positive regardless of their CD4 count, is a laudable WHO and UNAIDS model for the effective and efficient management of the HIV and AIDS pandemic, given its dual goals of improving health outcomes in people who are ignorant of their serostatus, and reducing HIV transmission. It is therefore indubitable that the test and treat strategy has objective potentials for saving lives of infected or potentially vulnerable persons. The obverse of the positives of the test and treat strategy is that resource-constrained countries need considerable human, logistic, and infrastructural readjustments, if they are to realistically and sustainably implement the aforementioned HIV and AIDS treatment model. It is in this light that this paper advocates for the putting in place of such mechanisms in Lesotho, in order to avoid the specter of poor retention of professional staff, non-sustainable supply chains of antiretroviral therapy drugs, accessible to the latter, ARV treatment defaulting, resistance to treatment, and long-term side effects of medications.

\section{Conflict of interest}

The authors declare no potential conflicts of interest with respect to the research, authorship, and/or publication of this article.

\section{References}

1. Kulkarni V. A turning point: The new World Health Organization guidelines for treatment of human immunodeficiency virus infection. Indian J Dermatol Venereol Leprol 2016; 82: 125-127.

2. Wilkin TJ, Gulick RM. When to start antiretroviral therapy? Clin Infect Dis 2008; 47: 1580-1586.

3. Jain V, Deeks SG. When to start antiretroviral therapy. Curr HIV/ AIDS Rep 2010; 7: 60-68.

4. Walensky PR, Hirsch MS. Age-Old Questions: When to Start Antiretroviral Therapy and in Whom? Clin Infect Dis 2015; 61: 1196-1198.

5. UNAIDS. 90-90-90 - An ambitious treatment target to help end the AIDS epidemic. JC2684, 2014 (English original, October 2014).

6. Evans D. HIV test and treat: challenges to overcome. Available at: www.aidsmeds.com/articles/hiv_test_treat_2581_20054.shtml (accessed 28.06.2011).

7. Gardner E, McLees M, Steiner J, et al. The spectrum of engagement in HIV care and its relevance to test-and-treat strategies for prevention of HIV Infection. Clin Infect Dis 2011; 52: 793-800.

8. Panel on Antiretroviral Guidelines for Adults and Adolescents. Guidelines for the use of antiretroviral agents in HIV-1-infected adults and adolescents. Department of Health and Human Services, Washington 2011; 27-40. Available at: www.aidsinfo.nih.gov/ ContentFiles/AdultandAdolescentGL.pdf (accessed 28.06.2011).

9. Attia S, Egger M, Muller M, et al. Sexual transmission of HIV according to viral load and antiretroviral therapy: systemic review and meta-analysis. AIDS 2009; 23: 1397-1404.

10. 10. Bemelmans R, Gelderblom GJ, Jonker P, et al. The potential of socially assistive robotics in care for elderly, a systematic review. In: Lamers MH, Verbeek FJ (eds.). Human-Robot Personal Relationships. HRPR 2010; 59: 83-89.

11. Lima VD, Hogg RS, Montaner JS, et al. Expanding HAART treatment to all currently eligible individuals under the 2008 IAS-USA Guidelines in British Columbia, Canada. PLoS One 2010; 5: e10991.

12. Metsch IR, Pereyra M, Messinger S, et al. HIV transmission risk behaviors among HIV-infected persons who are successfully linked to care. Clin Infect Dis 2008; 47: 577-584. 